Ab initio Molecular Dynamics Simulations of Water Under Static and Shock Compressed Conditions

Nir Goldman, Laurence E. Fried, Christopher J. Mundy, I-Feng. W. Kuo, Alessandro Curioni, Evan J. Reed

July 27,2007

American Physical Society conference on Shock Conditions of Condensed Matter Kona, HI, United States June 24, 2007 through June 29, 2007 
This document was prepared as an account of work sponsored by an agency of the United States Government. Neither the United States Government nor the University of California nor any of their employees, makes any warranty, express or implied, or assumes any legal liability or responsibility for the accuracy, completeness, or usefulness of any information, apparatus, product, or process disclosed, or represents that its use would not infringe privately owned rights. Reference herein to any specific commercial product, process, or service by trade name, trademark, manufacturer, or otherwise, does not necessarily constitute or imply its endorsement, recommendation, or favoring by the United States Government or the University of California. The views and opinions of authors expressed herein do not necessarily state or reflect those of the United States Government or the University of California, and shall not be used for advertising or product endorsement purposes. 


\title{
$A b$ initio Molecular Dynamics Simulations of Water Under Static and Shock Compressed Conditions
}

\author{
Nir Goldman*, Laurence E. Fried*, Christopher J. Mundy ${ }^{\dagger}$, I-F. William Kuo*, \\ Alessandro Curioni** and Evan J. Reed* \\ ${ }^{*}$ Lawrence Livermore National Laboratory, Chemistry Materials and Life Sciences Directorate, L-282, \\ Livermore, CA 94550 \\ ${ }^{\dagger}$ Pacific Northwest National Laboratory, Chemical and Materials Science Division, Richland, WA 99352 \\ ${ }^{* *}$ IBM Research, Zurich Research Laboratory, CH-8803, Ruesschlikon, Switzerland
}

\begin{abstract}
.
We report herein a series of $a b$ initio simulations of water under both static and shocked conditions. We have calculated the coherent $\mathrm{x}$-ray scattering intensity of several phases of water under high pressure, using $a b$ initio Density Functional Theory (DFT). We provide new atomic scattering form factors for water at extreme conditions, which take into account frequently neglected changes in ionic charge and electron delocalization. We have also simulated liquid water undergoing shock loading of velocities from $5-11 \mathrm{~km} / \mathrm{s}$ using the Multi-Scale Shock Technique (MSST). We show that Density Functional Theory (DFT) molecular dynamics results compare extremely well to experiments on the water shock Hugoniot.
\end{abstract}

Keywords: water, high pressure, high temperature, shock compression, ab initio Molecular Dynamics PACS: ??

\section{INTRODUCTION}

Detailed knowledge of the chemistry of water at extreme conditions (hundreds of GPa and thousands of Kelvin) is imperative to understanding earth and planetary sciences, and the chemical reactivity that occurs within such hot, compressed systems. Currently there is little scientific knowledge about reaction pathways and the nature of the molecular to nonmolecular transition at high temperature and pressure. It is not even clear if conventional chemical notions, such as covalent vs. ionic bonding, still hold at these conditions (e. g., [1]).

Despite recent technological advances, molecular and atomic scale information is still difficult to obtain experimentally, and theoretical studies are necessary. Phase diagram information is vital for equation of state determination; joint experimental and computational studies could lead to the discovery of new material properties. In one such example, re- cent results by Goncharov et al.[2] and Goldman et al.[3] have confirmed the presence of superionic water, an exotic phase wherein the oxygens have formed a stable body-centered-cubic (bcc) lattice and the hydrogens dissociate freely and diffuse at liquid-like rates[4]. Nonetheless, the high pressure phase diagram of water is not well defined, including the boundaries of the superionic phase, and between the molecular and the ionic liquids. In this work we present results on the structure of water under extreme conditions via $\mathrm{X}$-ray diffraction calculations, and equation of state data of water under shock compression.

\section{COMPUTATIONAL DETAILS}

For our static MD calculations we have used molecular dynamics trajectories generated for previous 
publications[5]. In summary, trajectories were generated by the CPMD code v.3.9.1, with the BLYP exchange-correlation functional, and TroullierMartins pseudo-potentials for both oxygen and hydrogen. A planewave cutoff of 120 Ry was employed to insure convergence of the pressure. The system size was $54 \mathrm{H}_{2} \mathrm{O}$ molecules, and all trajectories were run for 5-10 ps. The temperature was controlled by using Nosé-Hoover thermostats for all nuclear degrees of freedom. We chose a conservative value of 200 au for the fictitious electron mass, and a time step of $0.048 \mathrm{fs}$. Initial conditions were generated by relaxing an ice VII configuration at the density of interest, and heating to the temperature of interest via velocity scaling.

For our shock MD simulations, we used an optimized version of the CPMD code [6] for the Blue Gene/L supercomputer at Lawrence Livermore National Laboratory. We used Born-Oppenheimer Molecular Dynamics (labeled CPMD-BO) in conjunction with the Multi-Scale Shock Technique (MSST), discussed below, which requires only a small number of atoms in order to converge shock properties. MSST guarantees that the locus of thermodynamic states sampled remain on the Rayleigh line connecting ambient water to its shock Hugoniot. [7]. We performed six simulations with shock velocities from 5 to $11 \mathrm{~km} / \mathrm{s}$, each with a time step of $0.1935 \mathrm{fs}$. All simulations were started from the same configuration taken from an equilibrated CPMD simulation of $64 \mathrm{H}_{2} \mathrm{O}$ at ambient conditions. The shock compressed simulations were then run for $5-10$ ps. Results reported herein are averaged after the simulations have achieved compressed (within 1 ps in most cases)

\section{X-RAY SCATTERING}

The formalism for determination of $I(Q)$ and $g_{O O}(R)$ has been reviewed elsewhere[5, 8]. The x-ray scattering of an electron density generated from a condensed phase simulation trajectory can be calculated via Fourier Transform of the one electron density, $\rho(\mathbf{r})$. The coherent $\mathrm{x}$-ray scattering intensity from the $a b$ initio electron density, $I_{e l}(Q)$, was calculated via the QUICKSTEP code, which is part of the CP2K molecular dynamics suite $[8,5]$. Snapshots from our CPMD simulations were fed into the $\mathrm{CP} 2 \mathrm{~K}$ code in

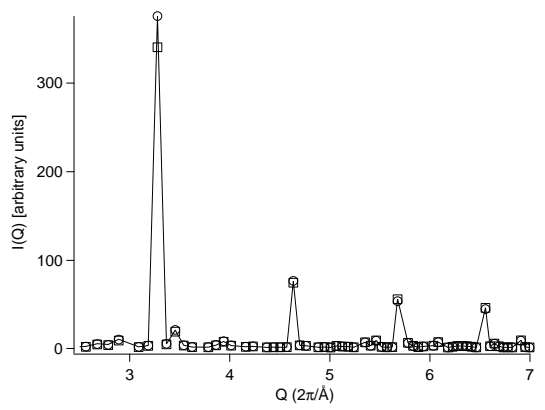

FIGURE 1. Comparison of computed $I_{e l}(Q)$ at $115 \mathrm{GPa}$ and $2000 \mathrm{~K}$. The squares corresponds to the result from the standard atomic form factors, and the solid line to the modified form factors with fitted values of $\alpha$ and $\delta$. The circles correspond to our DFT calculation of scattering.

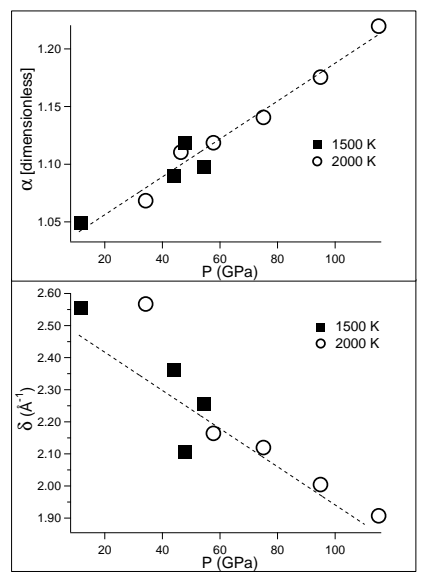

FIGURE 2. Results for 1500 and $2000 \mathrm{~K}$ of our best fit of $\alpha$ and $\delta$ for the modified form factors from Equation 1 . The dashed line corresponds to the linear regression result.

intervals of ca. $2.5-5$ fs. We used a basis set of TZVP for the oxygens and DZVP for the hydrogens, with the BLYP exchange-correlation functional. The smooth part of the electron charge density was was expanded to an energy cutoff of $130 \mathrm{Ry}$. All spectra were normalized according the modified algorithm presented in Ref. [5].

Frequently, the total electron density is approximated as a superposition of electron densities centered on individual atoms. Consequently, the total x-ray scattering intensity can be calculated via direct sum over atomic positions [5], using $f(Q)$ correspond to the atomic scattering factors. Standard val- 
ues of $f(Q)$ used in determination of the $I(Q)$ are calculated for the isolated atoms. However, this approximation is flawed in that as atoms come together to form covalent bonds, the charge density on each atom is clearly changed[8]. The effect is likely to be even more pronounced at high pressure-temperature conditions, where molecular bonding changes dramatically. With recent advances in ab initio codes [8], it has become possible but costly to directly calculate the total x-ray scattering intensity of a condensed phase simulation via Fourier Transform of the oneelectron density. We are thus in a unique position to determine more accurate form factors for high pressures and temperatures.

In order to modify the form factors for ambient water, Sorenson et al.[9] introduced the following:

$$
f^{\prime}(Q)=\left[1+(\alpha-1) \exp \left(-Q^{2} / 2 \delta^{2}\right)\right] f(Q),
$$

where $f^{\prime}(Q)$ is the modified atomic form factor, $f(Q)$ is the standard atomic form factor (same as above), and the $\alpha$ is a scaling factor representing the redistribution of charge, in this case mostly on the oxygens. The $\delta$ is a fitted parameter representing the valence electron delocalization caused by the formation of chemical bonds. We have used a LevenbergMarquardt non-linear least squares fitting routine[5] to fit the parameters of Eqn. 1 to our $I_{e l}(Q)$ data at 1500 and 2000 K. Our results for are shown in Figs. 1 and 2. Fig. 1 shows that our modified form factors can nearly exactly replicate the Fourier Transform of the one-electron density. In Fig. 2, the fact that $\alpha$ increases monotonically with pressure implies that the charge polarization between oxygen and hydrogen increases, as the superionic phase is formed[5]. In addition, the sharp monotonic decrease in fitted values of $\delta$ implies that the electron distribution about the oxygens is becoming less diffuse as a function of pressure. This implies that as the covalent $\mathrm{O}-\mathrm{H}$ interactions are weakening, consistent with calculated fleetingly short bond lifetimes (>10 fs)[3]. Our result allow for easy linear extrapolation to yield modified form factors experiments at high pressure and temperature

\section{SIMULATIONS OF SHOCK COMPRESSION}

The Multi-Scale Shock Technique (MSST) [10, 11] can dramatically reduce the number of parti- cles relative to non-equilibrium molecular dynamics (NEMD) methods, while guaranteeing that the simulation converges to the correct thermodynamic end state. The MSST [10] maintains the system on both the Rayleigh line $p-p_{0}=U^{2}\left(v_{0}-v\right) / v_{0}$, (where $U$ is the shock velocity) and the shock Hugoniot under condition of uniaxial strain of the computational cell. By regulating the strain rate of the computational cell, we guarantee that the $(P, T)$ thermodynamic states accessed during the shock simulation correspond to a steady macroscopic shock wave. This allows for much smaller system sizes to be used to achieve the same results as standard NonEquilibrium Molecular Dynamics simulations[11].

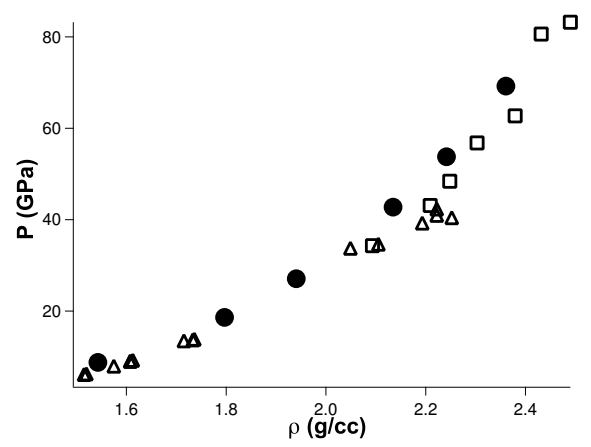

FIGURE 3. Plot of pressure vs density Hugoniot results. Our simulation results are shown with the solid black circles, the open triangles to experimental results from Walsh et al.[12], and the open squares to experimental results from Mitchell and Nellis[13].

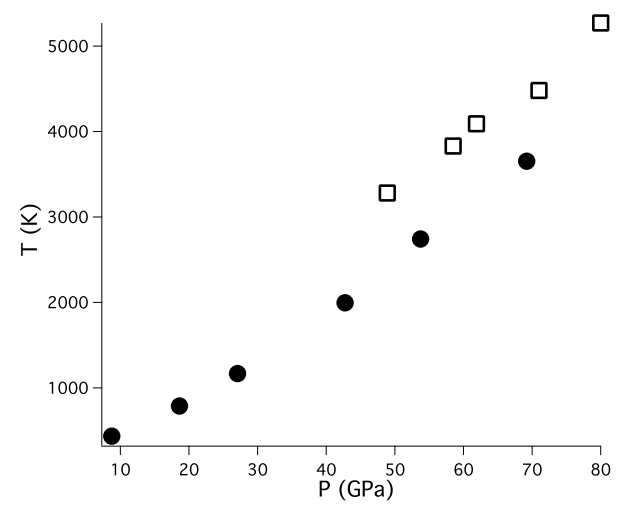

FIGURE 4. Plot of the temperature vs. pressure Hugoniot results. The solid black circles correspond to our CPMD-BO results, and the open squares to experimental results from Lyzenga et al. [14].

The CPMD-BO results for the shock hugoniot for 
water are shown in Figures 3 and 4. Our simulations produce excellent agreement with experimental results for the pressure vs. density equation of state of water. This provides excellent validation of DFT over a wide range of pressures (ca. $9-70$ $\mathrm{GPa}$ ). The agreement with experiment regarding the pressure-temperature shock Hugoniot is also remarkably good. Given the relative sparsity of temperature measurements in shock compression experiments, our temperature results are particularly relevant.

Mattsson et al. have shown the possible importance of including finite temperature effects (FT-DFT) in simulations of hot, compressed materials[15]. Given the accuracy with which we compare to experimental measurements of the shock Hugoniot as well as the conductivity, it is unlikely that such effects would be important for the thermodynamic states investigated in this work. Indeed, the inclusion of excited electronic states implies that our Born-Oppenheimer simulations would over estimate the temperature when in fact, Fig. 4 indicates the opposite. Conducitivty measurements by Chau et al. predict that electronic conduction is relevant in water only above $300 \mathrm{GPa}$ and $7000 \mathrm{~K}[16]$, well above the conditions studied in our work.

\section{CONCLUDING REMARKS}

We find that standard form factors with minor modifications (presented here) provide fairly accurate interpretation of $\mathrm{x}$-ray scattering data, even under extreme thermodynamic conditions. However, as the pressure and temperature are elevated to conditions where the nature of covalent bonding changes (e. g., superionic water), the standard form factors require larger adjustment. Our simulated spectra can provide excellent validation of the DFT under extreme conditions, since the $I(Q)$ allows for more direct comparison to experiment than more traditional methods (e. g., structure factor or radial distribution function). This could yield improvements in equation of state modeling of water at high pressures and temperatures.

Our DFT-MD simulations of water under shock loading show excellent agreement with experimental results of the shock Hugoniot states. Now that we have verified the ability of DFT to produce the cor- rect equation of state for water at high temperature and pressure, we can take steps to study chemical reactivity and species concentration behind the shock front. Future work will also include determination of proton conductivities.

\section{ACKNOWLEDGMENTS}

This work was performed under the auspices of the U.S. Department of Energy by University of California, Lawrence Livermore National Laboratory under Contract W-7405-Eng-48.

\section{REFERENCES}

1. Goldman, N., and Fried, L. E., J. Chem. Phys., 125, 044501 (2006).

2. Goncharov, A. F., Goldman, N., Fried, L. E., Crowhurst, J. C., Kuo, I.-F. W., Mundy, C. J., and Zaug, J. M., Phys. Rev. Lett., 94, 125508 (2005).

3. Goldman, N., Fried, L. E., Kuo, I.-F. W., and Mundy, C. J., Phys. Rev. Lett., 94, 217801 (2005).

4. Cavazzoni, C., Chiarotti, G. L., Scandolo, S., Tosatti, E., Bernasconi, M., and Parrinello, M., Science, 283, 44-46 (1999).

5. Goldman, N., and Fried, L. E., J. Chem. Phys., 126, 134505 (2007).

6. Hutter, J., and Curioni, A., ChemPhysChem, 6, 1788 (2005).

7. Reed, E., Fried, L. E., Manaa, M. R., and Joannopoulos, J. D., "A multi-scale approach to molecular dynamics simulations of shock waves," in Chemistry at Exteme Conditions, edited by M. Manaa, Elsevier, 2005.

8. Hura, G., Russo, D., Glaeser, R. M., Head-Gordon, T., Krack, M., and Parrinello, M., Phys. Chem. Chem. Phys., 5, 1981 (2003).

9. Sorenson, J. M., Hura, G., Glaeser, R. M., and Head-Gordon, T., J. Chem. Phys., 113, 9149 (2000).

10. Reed, E. J., Fried, L. E., and Joannopoulos, J. D., Phys. Rev. Lett., 90, 235503 (2003).

11. Reed, E. J., Fried, L. E., Henshaw, W. D., and Tarver, C. M., Phys. Rev. E, 74, 056706 (2006).

12. Walsh, J. M., and Rice, M. H., J. Chem. Phys., 26, 815 (1957).

13. Mitchell, A. C., and Nellis, W. J., J. Chem. Phys., 76, 6273 (1982).

14. Lyzenga, G. A., Ahrens, T. J., Nellis, W. J., and Mitchell, A. C., J. Chem. Phys., 76, 6282 (1982).

15. Mattsson, T. R., and Desjarlais, M. P., Phys. Rev. Lett., 97, 017801 (2006).

16. Chau, R., Mitchell, A. C., Minich, R. W., and Nellis, W. J., J. Chem. Phys, 114, 1361 - 1365 (2001). 